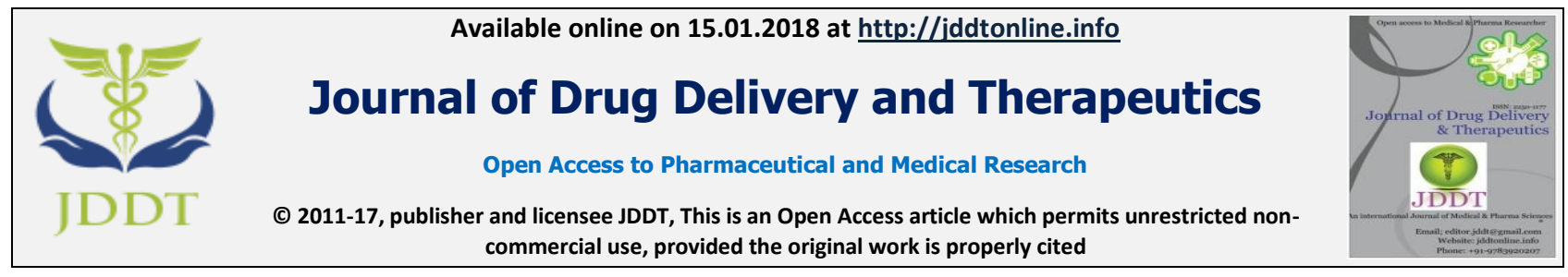

Open $\odot$ Access

Research Article

\title{
HPTLC METHOD DEVELOPMENT AND VALIDATION FOR ESTIMATION OF CINNAMALDEHYDE IN POLYHERBAL FORMULATION WITH ANTI- DIABETIC PROPERTY
}

\author{
Satyaendra K. Shrivastava ${ }^{1}$, P.K. Dubey ${ }^{1}$, Pankaj Sharma ${ }^{2}$, B. Shrivastava ${ }^{2}$ \\ ${ }^{1}$ Swami Vivekanand College of Pharmacy, Khandwa Road, Indore (M.P.), India \\ ${ }^{2}$ School of Pharmaceutical Sciences, Jaipur National University, Jaipur (Rajasthan), India
}

\section{ABSTRACT}

In this research article HPTLC method for the estimation of cinnamaldehyde content in cinnamomum zeylanicum, laboratory batches (MC-I, MC-II, MC-III) and marketed formulation (MCM) was developed. In this method, precoated Silica Gel $\mathrm{F}_{254}$ plates were used as stationary phase and Toluene: ethyl acetate: formic acid $(8: 1: 1 \mathrm{v} / \mathrm{v})$ as mobile phase. Developed chromatogram was scanned at 295 $\mathrm{nm}$, the wavelength of maximum absorption for cinnamaldehyde. The aptness of developed HPTLC method for estimation of cinnamaldehyde was established by validating it as per the ICH guidelines. The developed method has been successfully used for the estimation of cinnamaldehyde in polyherbal anti-diabetic formulation.

Keywords: Polyherbal formulation, Cinnamaldehyde, Cinnamomum zeylanicum, HPTLC.

Article Info: Received 11 Nov, 2017; Review Completed 08 Jan, 2018; Accepted 09 Jan, 2018; Available online 15 Jan, 2018

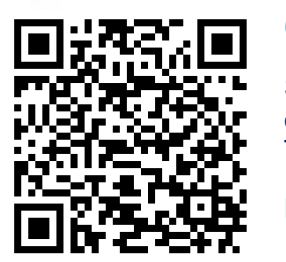

Cite this article as:

Shrivastava SK, Dubey PK, Sharma P, Shrivastava B, HPTLC method development and validation for estimation of cinnamaldehyde in polyherbal formulation with anti-diabetic property, Journal of Drug Delivery and Therapeutics. 2018; 8(1):70-73

DOI: http://dx.doi.org/10.22270/jddt.v8i1.1553

\section{*Address for Correspondence}

Satyaendra K. Shrivastava, Swami Vivekanand College of Pharmacy, Khandwa Road, Indore (M.P.), India. E-mail: shrivastavapharma@gmail.com, skshrivastava@svcp.ac.in

\section{INTRODUCTION}

Being resurrecting of interest in natural drugs, especially plants derived, started in the last few decades mainly because of widespread belief that green medicines are healthier and safer than the synthetic once 1 . Standardization of herbal materials and their formulations is essential in order to assess quality of the drugs. The World Health Organization (WHO) collaborates and assists health ministries in establishing mechanisms for the introduction of traditional plant medicines in assessing safety and efficacy ${ }^{2}$. They also appreciated the importance of medicinal plants for public health care in developing nations and has evolved guidelines to support the member states in their efforts to formulate national policies on traditional medicine and to study their potential usefulness including evaluation, safety and efficacy ${ }^{3}$. A qualitative method provides information about the identity of sample, whereas, a quantitative method provides numerical information as to the relative amount of one or more of these components. High-performance thin-layer chromatography (HPTLC) is still increasingly finding its way in pharmaceutical analysis in some parts of the world. The technique achieves for given applications a precision and trueness comparable to high performance liquid chromatography (HPLC). It also allows simultaneous estimation of several samples utilising only a small quantity of a mobile phase, hence minimising the analysis time and cost ${ }^{4}$.

A well known polyherbal formulation for diabetes mellitus was selected for study which comprises of some medicinally important plants, Gymnea sylvestre (leaves), Eugenia jamboloma (seeds), Aegle marmelos (leaves), Azadirachta indica (leaves), Cinamomum 
zeylanicum (leaves), Sphaeranthus indicus (flower), Momordica charantia (fruits), Trivang bhasma and Shilajeet and excipients. Three laboratory batches of capsule were prepared and named as MC-I, MC-II, MCIII and marketed formulation MCM was purchased from local pharmacy store, Indore.

On the basis of review of literature a few analytical techniques have been reported for the analysis of cinnamaldehyde is available for its estimation in polyherbal formulations. The main aim of this study was to develop and validate HPTLC method for estimation of cinnamaldehyde in selected polyherbal capsule. The validation was done as per ICH guidelines ${ }^{5}$.

\section{MATERIALS AND METHODS}

The HPTLC fingerprinting method was developed for Cinamomum zeylanicum, each laboratory batch (MC-I, MC-II, MC-III) and marketed formulation (MCM) via estimation of cinnamaldehyde by using following experimental techniques. All chemicals and reagents used were of analytical grade and were purchased from Hi-Media, India.

\section{Instrumentation and chromatographic conditions:}

Spotting device: Linomat V Automatic Sample Spotter; CAMAG (Muttenz, Switzerland)

Syringe : $100 \mu \mathrm{L}$ Hamilton (Bonaduz, Switzerland)

TLC Chamber: Glass twin trough chamber $(20 \times 10 \times 4 \mathrm{~cm})$

CAMAG Densitometer: TLC Scanner 3 linked to Win Cats software V.4.06; CAMAG

HPTLC plates: $10 \times 10 \mathrm{~cm}, 0.2 \mathrm{~mm}$ thickness precoated with silica gel $60 \mathrm{~F}_{254}$

Temperature: $25 \pm 2{ }^{\circ} \mathrm{C}$, relative humidity $40 \%$

Solvent system: Toluene: ethyl acetate: formic acid $(8: 1: 1 \mathrm{v} / \mathrm{v})$

Detection Wavelength: $295 \mathrm{~nm}$

Scanning Speed: $20 \mathrm{~mm} / \mathrm{s}$

Source: Deuterium lamp

\section{Standard solutions of cinnamaldehyde}

The stock solution of cinnamaldehyde was prepared by dissolving $10 \mathrm{mg}$ of cinnamaldehyde in $100 \mathrm{ml}$ of methanol. The final solution contained $100 \mu \mathrm{g}$ of the cinnamaldehyde per $\mathrm{ml}$ of the solution.

\section{Calibration curve of cinnamaldehyde}

A stock solution of cinnamaldehyde $\left(100 \mu \mathrm{gmL}^{-1}\right)$ was prepared in methanol. Different volumes of stock solution were spotted on the TLC plate to obtain concentrations of $100-600 \mathrm{ng} \operatorname{spot}^{-1}$ of cinnamaldehyde, respectively. The data of peak areas plotted against the corresponding concentrations were treated by least-square regression analysis method validation (Figure 1).

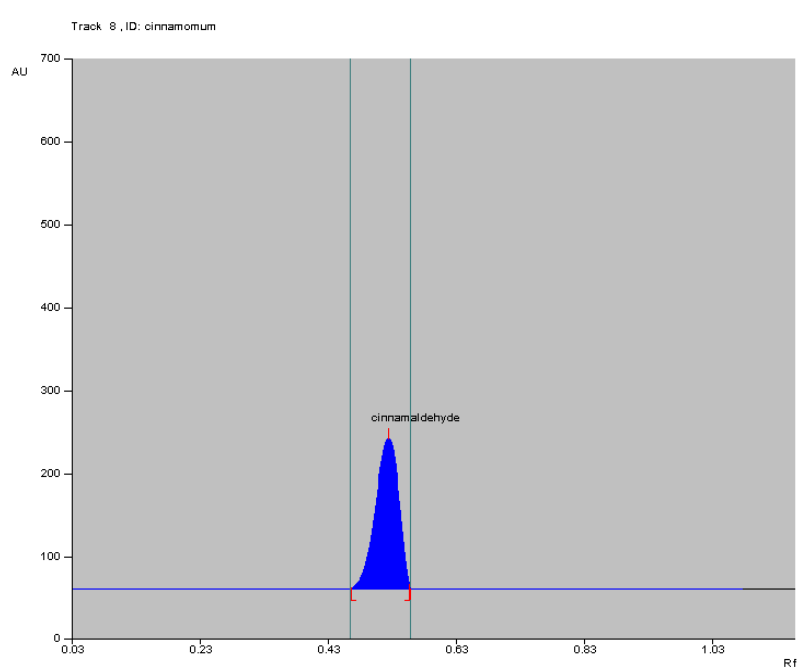

Figure 1: HPTLC chromatogram of cinnamaldehyde

\section{Method validation}

The method was validated for precision, accuracy, limit of detection, limit of quantification, robustness, ruggedness \& specificity of sample application. The results were containing in table 3 .

\section{Precision}

Repeatability of the sample application and measurement of peak area were carried out using six replicates of the same spot and was expressed in terms of percent relative standard deviation (\% R.S.D.). The intra and inter-day variation for the determination of cinnamaldehyde was carried at three different concentration levels of $100,300,600 \mathrm{ng} \operatorname{spot}^{-1}$ (Table $1)$.

Table 1: Intra- and inter-day precision of HPTLC method (n=6)

\begin{tabular}{|l|l|c|c|c|c|}
\hline Principle constituent & Amount & \multicolumn{2}{|c|}{ Intra -day precision } & \multicolumn{2}{c|}{ Inter-day precision } \\
\cline { 2 - 6 } & (ng/spot) & S.D. of area & RSD \% & S.D. of area & RSD \% \\
\hline \multirow{3}{*}{ Cinnamaldehyde } & 100 & 0.113 & 0.0038 & 0.403 & 0.0135 \\
\cline { 2 - 6 } & 300 & 0.112 & 0.0019 & 0.667 & 0.0118 \\
\cline { 2 - 6 } & 600 & 0.091 & 0.0010 & 0.309 & 0.0036 \\
\hline
\end{tabular}

\section{Limit of detection (LOD) \& Limit of quantification $(\mathrm{LOQ})$}

LOD was determined based on the lowest concentration detected by instrument in the sample. LOQ was determined based on the lowest concentration quantified by the instrument in the sample (LOQ $=5 \times$ LOD). In order to estimate the limit of detection (LOD) and limit of quantification (LOQ), blank methanol was spotted six times LOD was considered as 3:1 and LOQ as 10:1. LOD and LOQ were experimentally verified by diluting 
the known concentrations of cinnamaldehyde until the average responses were approximately 3 or 10 times the standard deviation of the responses for six replicate determinations.

\section{Robustness}

By introducing small changes in the mobile phase composition, mobile phase volume, duration of mobile phase saturation and activation of pre washed TLC plates with water; the effects on the results were examined. Robustness of the method was done in triplicate at a concentration level of $600 \mathrm{ng} \mathrm{spot}^{-1}$ for cinnamaldehyde and the \% R.S.D of peak areas was calculated.

\section{Ruggedness}

A solution of concentration $600 \mathrm{ng} \mathrm{spot}^{-1}$ was prepared and analyzed on day 0 and after 6, 12, 24, 48 and $72 \mathrm{~h}$. Data were treated for \% RSD to assess ruggedness of the method for cinnamaldehyde.

\section{Specificity}

The specificity of the method was confirmed by analyzing the standard drugs and samples. The spot for ellagic acid in the sample was confirmed by comparing the $R_{f}$ values and spectra of the spot with that of the standard. The peak purity of the cinnamaldehyde was assessed by comparing the spectra at three different levels.

\section{Recovery}

The recovery was determined by the standard addition technique. The pre-analyzed samples were spiked with extra 50,100 and $150 \%$ of the standard cinnamaldehyde and the mixtures were reanalyzed by the proposed method. The experiment was conducted six times. This was done to check for the recovery of the cinnamaldehyde at different levels in the formulations (Table 2).

Table 2: \% Recovery for cinnamaldehyde

\begin{tabular}{|c|c|c|c|c|}
\hline Excess drug added to the analyte (\%) & Conc. found & S. D. & Recovery (\%) & R.S.D. (\%) \\
\hline 50 & 148.73 & 0.9237 & 99.15 & 0.621085 \\
\hline 100 & 198.13 & 1.3012 & 99.06 & 0.656771 \\
\hline 150 & 248.90 & 0.8888 & 99.56 & 0.357099 \\
\hline
\end{tabular}

Table 3: HPTLC validation Parameters of cinnamaldehyde

\begin{tabular}{|l|l|}
\hline Parameters & Data of Cinnamaldehyde \\
\hline Linearity range & $100-600 \mathrm{ng} / \mathrm{spot}$ \\
\hline Correlation coefficient & 0.993 \\
\hline Limit of detection $(\mu \mathrm{g} / \mathrm{ml})$ & 18.34 \\
\hline Limit of quantitation $(\mu \mathrm{g} / \mathrm{ml})$ & 56.28 \\
\hline Recovery $(\mathrm{n}=3)$ & $99.26 \%$ \\
\hline $\begin{array}{l}\text { Precision }(\text { R.S.D. \%) } \\
\text { Repeatability of application }(\mathrm{n}=6)\end{array}$ & 0.00055 \\
\hline $\begin{array}{l}\text { Inter-day }(\mathrm{n}=6) \\
\text { Intra-day }(\mathrm{n}=6)\end{array}$ & 0.00223 \\
\hline Robustness & 0.00963 \\
\hline Specificity & Robust \\
\hline & Specific \\
\hline
\end{tabular}

Estimation of cinnamaldehyde in crude drug and formulations

Accurately weighed $1 \mathrm{gm}$ cinnamon bark was ground to pass through a 1-mm screen and the powder obtained was extracted at room temperature by constant percolation with ether. The solvent was evaporated using a rotator evaporator, under vacuum, at $35^{\circ} \mathrm{C}$. This extract was centrifuged at 2,000 rpm for $15 \mathrm{~min}$ at $30^{\circ} \mathrm{C}$. The filtered solution was applied on the TLC plate followed by development and scanning. The analysis was repeated in triplicate. The same procedure was performed for each batch of formulations and separately powdered drug. A single spot at $\mathrm{R}_{\mathrm{f}}=0.52$ was observed in the chromatogram of the cinnamaldehyde, along with other components. The total cinnamaldehyde content in cinnamomum zeylanicum, laboratory batches (MC-I, MC-II, MC-III) and marketed formulation (MCM) were reported in table 4.

Table 4: HPTLC estimation of cinnamaldehyde in crude drug and formulations

\begin{tabular}{|c|l|l|l|}
\hline S. No. & \multicolumn{1}{|c|}{ Name } & Cinnamaldehyde content (\%w/w) & Confidence level (95\%) \\
\hline & Cinamomum zeylanicum & $0.4850 \pm 0.025$ & \pm 0.143 \\
\hline & MC-I & $0.0062 \pm 0.064$ & \pm 0.124 \\
\hline & MC-II & $0.0065 \pm 0.045$ & \pm 0.145 \\
\hline & MC-III & $0.0066 \pm 0.074$ & \pm 0.126 \\
\hline & MCM & $0.0054 \pm 0.035$ & \pm 0.141 \\
\hline
\end{tabular}




\section{CONCLUSION}

The developed HPTLC technique is precise, accurate and robust for the determination of cinnamaldehyde in formulated and marketed formulation. Statistical analysis proves that the method is reproducible for the analysis of cinnamaldehyde. The content of cinnamaldehyde in marketed polyherbal capsule formulation is comparable to that of laboratory

\section{REFERENCES}

1. Govil GN, Singh VK: Recent Progress in Medicinal Plants: Standardization of herbal/Ayurvedic formulations, Studium Press LLC Publisher, 2009; 24:46.

2. Rose EN, Nmedo KP and Samal PK. J. Pharm. Tech., 2008; $1: 310-312$.

3. Organisation Mondiale De La Sante.: Quality control methods for medicinal plant materials, 559, (1) Original English, World Health Organisation, 1992:159. formulations. Therefore, this method can be successfully used for the routine analysis of cinnamaldehyde for standardization and quality control of pharmaceutical products.

\section{ACKNOWLEDGEMENT}

Authors gratefully acknowledge Institute of Pharmacy, Pt. Ravi Shankar Shukla University, Raipur, for assistance in HPTLC work.

4. Sethi PD. HPTLC: High Performance Thin Layer Chromatography, CBS Publishers and Distributors, New Delhi, India, 1996; 11-17.

5. ICH. (2005). Validation of analytical procedures: text and methodology. ICH-Q2 (R1), International Conference on Harmonization: Geneva. 